\title{
RETRACTED ARTICLE: A Case Series of Four Patients With Clinically Significant Radiomicrosphere Pneumonitis After Yttrium-90 Radioembolization from the Perspective of Lung Dosimetry
}

\author{
Yung Hsiang Kao • Butch M. Magsombol • Angela Takano • \\ Limin Wijaya $\cdot$ Kiang Hiong Tay $\cdot$ Pierce K. H. Chow \\ Anthony S. W. Goh • David C. E. Ng
}

Received: 8 July 2014 /Revised: 16 October 2014 / Accepted: 27 October 2014 / Published online: 11 November 2014

(C) Korean Society of Nuclear Medicine 2015

Dr. Yung Hsiang Kao, the first author and corresponding author, has retracted this case report due to honest error. The methodology used for this case report was unsuitable for the reporting of incidence rates and therefore does not accurately portray the disease incidence at the institution concerned.
Y. H. Kao • B. M. Magsombol • A. S. W. Goh • D. C. E. Ng Department of Nuclear Medicine and PET, Singapore General Hospital, Outram Road, Singapore 169608, Singapore

\section{Y. H. Kao $(\bowtie)$}

Department of Nuclear Medicine, Austin Hospital, Level 1, Harold Stokes Building, 145 Studley Road, Heidelberg, Melbourne, Victoria 3084, Australia

e-mail: yung.h.kao@gmail.com

\section{A. Takano}

Department of Pathology, Singapore General Hospital, Outram Road, Singapore 169608, Singapore

\section{Wijaya}

Department of Infectious Disease, Singapore General Hospital, Outram Road, Singapore 169608, Singapore

\author{
K. H. Tay \\ Department of Diagnostic Radiology, Singapore General Hospital, \\ Outram Road, Singapore 169608, Singapore \\ P. K. H. Chow \\ Department of General Surgery, Singapore General Hospital, Outram \\ Road, Singapore 169608, Singapore \\ P. K. H. Chow \\ Department of Surgical Oncology, National Cancer Centre \\ Singapore, 11 Hospital Drive, Singapore 169610, Singapore \\ P. K. H. Chow \\ Office of Clinical Sciences, Duke-National University of Singapore \\ Graduate Medical School, 8 College Rd, Singapore 169857, \\ Singapore
}

